\title{
Hybride Formen urbaner Produktion durch Digitalisierung? Trends und Beispiele aus Nordrhein-Westfalen
}

\section{Hybrid forms of urban production through digitalisation? Trends and cases from North Rhine-Westphalia}

https://doi.org/10.2478/rara-2020-0013

Eingegangen: 30. September 2019 / Angenommen: 20. März 2020

\begin{abstract}
Kurzfassung: Dieser Beitrag knüpft an aktuelle Diskurse zu Digitalisierungsprozessen und Raumentwicklung an. Es wird untersucht, inwiefern durch digitalisierte Fertigungsmethoden (wie Industrie-4.0-Anwendungen) neue Formen urbaner Produktion entstehen können. Aufbauend auf theoretisch-konzeptionellen Überlegungen wurden empirische Erkenntnisse durch 41 Experteninterviews in acht industriell geprägten Städten Nordrhein-Westfalens gewonnen. Die Ergebnisse zeigen, dass durch die gewerbliche Anwendung neuer digitaler Fertigungsverfahren neuartige Formen hybrider digitaler urbaner Produktion entstehen, die spezifischen problemzentrierten Wertschöpfungslogiken unterliegen. Der Beitrag entwickelt ein präziseres Verständnis dieser Wertschöpfungsprozesse und der Standorteinbettung hybrider digitaler urbaner Produktion.
\end{abstract}

Schlüsselwörter: Urbane Produktion, Digitalisierung, hybride Produktion, Problemlösungszyklus, Nordrhein-Westfalen

Abstract: Building on current debates on digitalisation and spatial development processes, this paper aims to explore to what extent digitally enhanced production technologies, such as Industry 4.0, enable the emergence of new forms of urban production. Based on theoretical and conceptual considerations, empirical insights are derived from 41 interviews conducted with experts in eight industrial cities in the German state of North Rhine-Westphalia. The results indicate that the commercial application of new digital production technologies instigates novel forms of hybrid digital urban manufacturing which change patterns of previous value creation. The paper develops a more precise understanding of these value creation processes and the locational embeddedness of hybrid digital urban manufacturing.

Keywords: Urban production, Digital transformation, Hybrid production, Problem-solving cycle, North Rhine-Westphalia

\footnotetext{
*Corresponding author: Hans-Christian Busch, Universität zu Köln, Wirtschafts- und Sozialgeographisches Institut, AlbertusMagnus-Platz, 50923 Köln, Deutschland, E-mail: hans-christian.busch@wiso.uni-koeln.de

Caroline Mühl, Prof. Dr. Martina Fromhold-Eisebith, Rheinisch-Westfälische Technische Hochschule Aachen, Geographisches Institut, Wüllnerstraße 5b, 52062 Aachen, Deutschland

Prof. Dr. Martina Fuchs, Universität zu Köln, Wirtschafts- und Sozialgeographisches Institut, Albertus-Magnus-Platz, 50923 Köln, Deutschland
} 


\section{Einleitung}

In wissenschaftlichen und politischen Debatten zeigt sich ein wachsendes Interesse an den gesellschaftlichen Auswirkungen der Digitalisierung. Auch deren Implikationen für städtische Ökonomien, speziell produzierende Unternehmen, erfahren aktuell eine hohe Aufmerksamkeit (Läpple 2016: 23 ff.; Reynolds 2017: 26; Gornig/Werwatz 2018: 1006 ff.). Das hierzu geprägte Schlagwort "Industrie 4.0" hat sich national wie international als Sammelbegriff der Anwendung digitaler Innovationen in der industriell-gewerblichen Produktion durchgesetzt (vgl. Kagermann/Ander//Gausemeier et al. 2016). Im Zuge einer weiter gefassten, nicht nur ingenieurwissenschaftlichen Begriffsbestimmung lässt sich Industrie 4.0 als Bündel diverser produktionstechnologischer Innovationen begreifen, die sich maßgeblich auf neue digitale Steuerungs- und Koordinationsmöglichkeiten stützen. Dies umfasst die weitreichende Vernetzung materieller, über das "Internet der Dinge“ (IoT, Internet of Things) bzw. Cyber-Physische Systeme gesteuerte Produktionsprozesse (Fromhold-Eisebith 2017: 3 ff.) ebenso wie neue additive oder subtraktive Fertigungsverfahren, wie 3D-Druck- oder Laser-Schneideverfahren (Gress/ Kalafsky 2015: 43). Bislang stellt Industrie 4.0 allerdings eine erst teilweise betrieblich realisierte technologiepolitische Leitvorstellung dar (Hirsch-Kreinsen 2016: 5). Dies erschwert deren empirische Fassbarkeit, nicht zuletzt aufgrund der Vielzahl aktuell verbreiteter ,4.0'-Derivate.

Mit den neuen Potenzialen digitaler Produktionstechnologien verbinden Akteure aus Politik, Forschung und Verbänden große Chancen zur Revitalisierung von Industrie und Gewerbe im städtischen Raum, ausgedrückt im Begriff der „Urbanen Produktion“ (Spath 2014: 65; Stiehm 2017: 3). Nach langjährigen Tendenzen der Abwanderung ehemals innerstädtischer Produktionsunternehmen in das Umland bzw. sogar des Offshoring ins ferne Ausland entstehen nun Visionen einer Aufwertung städtischer Standorte für Industrie und Handwerk. Moderne Produzenten seien - so die optimistische Sicht - umweltverträglicher und von nur geringen Flächennutzungskonflikten mit dem Wohnumfeld geprägt, assoziiert mit einer "Stadt der kurzen Wege" oder einer "Stadt der Zukunft" (Lentes 2017: 48; Matt/Orzes/Rauch et al. 2018: 4).

Inwiefern Industrie 4.0 wirklich neue Chancen für Gewerbe im urbanen Raum schafft, ist bislang empirisch jedoch noch kaum untersucht worden. Wir wollen mit unserer Untersuchung vor allem zum besseren empirischen Verständnis der Wirkungen von neuen digitalen Produktionstechnologien auf urbane Produktion beitra- gen, gemäß der Leitfrage: Inwiefern können durch digitalisierte Fertigungsverfahren (wie Industrie-4.0-Anwendungen) neue Formen industrieller und handwerklicher Produktion im urbanen Raum entstehen? Im Zuge eines hierauf ausgerichteten explorativ-qualitativen Untersuchungsansatzes haben wir im Zeitraum 2017 bis 2018 empirische Forschungsarbeiten (unter anderem 41 leitfadengestützte Experteninterviews) in acht Stadtregionen Nordrhein-Westfalens durchgeführt.

Nachfolgend stellt Kapitel 2 den Forschungsstand zu Auswirkungen der Digitalisierung auf die Produktion im städtischen Umfeld vor. Anschließend beschreibt Kapitel 3 den konzeptionellen Rahmen unserer Untersuchung. Kapitel 4 geht näher auf das Untersuchungsgebiet, das explorativ-qualitative Forschungsdesign und die für Datenerhebung und -analyse angewandte Methodik ein. Die Ergebnisse unserer empirischen Arbeiten präsentiert Kapitel 5. In Kapitel 6 diskutieren und bewerten wir die Resultate im Lichte der wissenschaftlichen Debatte und ziehen in Kapitel 7 ein Fazit.

\section{Stand der Forschung: Digitalisierung und urbane Produktion}

Die wissenschaftliche Debatte um Digitalisierung und Industrie-4.0 im Kontext städtischer Ökonomien ist Teil des Diskurses über Digitalisierungsprozesse und Raumentwicklung, der bereits in den 1980er-Jahren einsetzte. Das Interesse richtete sich auf räumliche Dimensionen der Transformation zur Informationsgesellschaft (Leborgne/Lipietz 1988: 263), auf die Rolle der Mikroelektronik in der internationalen Arbeitsteilung (Ernst 1985: 334; Hoffman 1985: 379) und den Beitrag dieser neuen Technologien zur Stadtentwicklung (Henckel/ Nopper/Rauch 1984: 10). Im Zentrum des Diskurses stand dabei die Frage, inwiefern Digitalisierung eher zu einer Zentralisierung oder Dezentralisierung (global und regional) von Wirtschaft und Bevölkerung beiträgt. Bezogen auf die Stadtentwicklung ging es dabei um Aspekte wie zunehmende Suburbanisierung sowie die Steigerung der Qualität von Arbeiten und Leben in der Innenstadt und in citynahen Räumen.

In der mit der Jahrtausendwende aufkommenden "Geographie des Internetzeitalters" (Leamer/Storper 2001: 641) stellten sich weitere auf den Stadtraum bezogene Erkenntnisse ein: Zwar wächst die Ortsunabhängigkeit von Betrieben vor allem bei Dienstleistungsunternehmen (Adam 2001: 170), doch wird die räumliche 
Nähe zwischen Akteuren - insbesondere in Städten nach wie vor als hoch relevant eingeschätzt (Moriset/ Malecki 2009: 271). Face-to-face-Kontakte würden folglich nicht durch elektronische Fernkommunikation ersetzt (Leamer/Storper 2001: 652). So zeigen van Winden, van den Berg, Carvalho et al. (2011: 358), dass städtische Warenproduktion vor allem „kleiner, sauberer und wissensintensiver" geworden ist. Statt großräumiger Gewerbeflächen für Fabrikanlagen benötigt die sich wandelnde urbane Produktion insbesondere hoch qualifizierte Beschäftigte, internationale Anbindungen sowie die Nachbarschaft zu Forschungsinstituten (van Winden/ van den Berg/Carvalho et al. 2011: 358; vgl. auch Musil/ Eder 2017: 94).

Die aktuelle Debatte um Digitalisierung und Industrie 4.0 betont den Aspekt der (Re-)Konzentration digitaler Produktion in städtischen Räumen - oder argumentiert zumindest gegen eine (weitere) Abwanderung von Industrien. Gemäß dem Motto „Produktion zurück in die Stadt" (Läpple 2016: 22) gehen verschiedene Akteure davon aus, dass Industrie 4.0 eine "Stadt der kurzen Wege" (Lentes 2017: 48) ermöglicht, die industrielle und handwerkliche Warenproduktion "stadtverträglich“ in das urbane Umfeld integriert (Matt/Orzes/Rauch et al. 2018: 4). Neben langjährig im Stadtraum bestehenden Produktionsunternehmen ergibt sich somit potenziell ein erweitertes Feld für digitale urbane Produktion, die insbesondere neue digitalisierte, additive und subtraktive Produktionstechnologien im weiteren Bereich von Industrie 4.0 nutzt, z. B. 3D-Druck, Laser-Schneideverfahren oder Mehr-Achs-CNC-Fräsverfahren.

Das Wirkungsgefüge dieser emergenten digitalen urbanen Produktion wird jedoch erst seit Kurzem wissenschaftlich untersucht (Stiehm 2017: 64 ff.). In diesem Rahmen werden für solche Produktionsaktivitäten verschiedene Erfordernisse postuliert: Kleine Losgrößen, die durch innovative Industrie-4.0-Fertigungsverfahren realisiert werden, individualisierte Produkte, die unter hohem Einbezug der Endkunden erstellt werden, schadstoffarme und emissionsfreie Produktionsprozesse, neue Formen der Prozess- und Arbeitsplatzgestaltung sowie eine Produktion auf kleinen Flächen (Stiehm 2017: $64 \mathrm{f})$. Basis erfolgreicher digitaler urbaner Produktion ist zudem das Angebot spezialisierter Facharbeiterinnen/ Facharbeiter und eine leistungsfähige digitale Infrastruktur im urbanen Raum, die eine Grundvoraussetzung der Nutzung digitalisierter Produktionstechnologien bildet (Mühl/Busch/Fromhold-Eisebith et al. 2019: $41 \mathrm{ff}$.). Dennoch weisen die bisherigen Untersuchungen eine definitorische Unschärfe in Bezug auf digitale urbane Produktion auf.
Mit der Idee einer (nachhaltigen) urbanen Produktion wird (wieder) vor allem die Einbettung in die „urbane Wissensökonomie" verbunden (Musil/Eder 2017: 93). Die „zunehmende Innovations- und Wissensorientierung der Industrieproduktion" (Musil/Eder 2017: 94) macht den urbanen Standort zum zukünftig wichtigsten Umfeld für gewerbliche Warenproduktion (Juraschek/Vossen/ Hoffschröer et al. 2018: 195). Die Annahme, dass die wissensintensive digitale urbane Produktion grundsätzlich vom städtischen Kontext profitiert, bestätigen Überlegungen von Florida, Adler und Mellander (2017: 93), denen zufolge speziell radikale Innovationen nicht bloß in Städten stattfinden, sondern das städtische Umfeld sogar als notwendige Voraussetzung zwingend erfordern. So betont Reynolds (2017: 26) die systemische Rolle urbaner Produktion, die im Rahmen der Digitalisierung vermehrte Aufmerksamkeit verlangt: „urban manufacturing in the future will be understood and appreciated more for the role it plays within the innovation ecosystem [...]. While the city continues to offer key strategic locational advantages, it is its role as a source of talent and innovation that will drive manufacturing to locate in and near cities" (vgl. auch Reynolds/Uygun 2018: 178).

Im Zuge des systemischen digitalen Wandels im urbanen Raum werden beispielsweise sogenannte "offene Werkstätten" als neue Produktionsformen der Stadt diskutiert (Lange 2017: 33; Lange/Bürkner 2018a: 99). Was die Wertschöpfungsprozesse in solchen Werkstätten betrifft, liegen dort weniger ,klassisch“ linear verlaufende Wertschöpfungsketten, sondern eher problembasierte, zirkulär verlaufende Value-Shop-Ansätze vor (Stabell/Fjeldstad 1998: 420 ff.). Innerhalb von Value Shops wird die Wertschöpfungsaktivität individuell an das zu lösende (Kunden-)Problem angepasst, wobei die Arbeitsprozesse und deren Abfolge auf den spezifischen Problemfall ausgerichtet werden (Bathelt/Glückler 2018: 183). Diese Darstellung des Wertschöpfungsprozesses hat insbesondere urbane wissensintensive Dienstleistungen bereits treffend erklären können (Glückler 2007: 950). Denn hier erfolgen Problemstellung und Problemlösung tendenziell am selben Ort, der deshalb - in Anlehnung an den englischen Begriff Workshop, das heißt die Werkstatt eines Handwerksmeisters - auch als „Wertstätte“ übersetzt wird (Handke 2014: 209 ff.).

Anders als bei der linear-sequenziellen Wertschöpfungskette prägt ein zirkulär und iterativ verlaufender ,Problemlösungskreislauf‘ den Value-Shop-Ansatz. In der Ursprungskonzeption umfasst er die fünf sich wiederholenden Schritte (1) Problemdefinition, (2) Ermittlung potenzieller Problemlösungsalternativen, (3) Lösungsauswahl aus den zuvor definierten Lösungsalternativen, 
(4) Durchführung der ausgewählten Lösungsalternative und (5) Kontrolle und Evaluation der durchgeführten Alternative gegenüber dem zu lösenden Anfangszustand des definierten Problems (Stabell/Fjeldstad 1998: 423; Handke 2014: 238). Somit beruht das Modell nicht auf vertikalen Verflechtungen, sondern erlaubt eine flexible, problemspezifische Ressourcenallokation und Aktivitätsabfolge (Bathelt/Glückler 2018: 183). Der Zyklus ist nach Schritt (5) entweder beendet (das heißt, das anfängliche Problem wurde gelöst) oder ein neuer Durchlauf wird initiiert, der gegebenenfalls Anpassungen des Lösungsprozesses erfordert (Stabell/Fjeldstad 1998: 424).

Während die zirkuläre Form der Problemlösung und Wertkreation zunächst vor allem für urbane Produktion im Bereich des Maker Movement' untersucht wurde (Lange 2017: 35), sind auch Bezüge zum urban produzierenden Gewerbe sowie Handwerk erkennbar. Das Handwerk ist traditionell im urbanen Raum angesiedelt und besetzt eine Schnittstellenfunktion zwischen Dienstleistung und Produktion und wird aufgrund dieser hybriden Eigenschaften als Zukunftsmodell urbaner Produktion betrachtet (Piegeler/Spars 2019: 14). Vor allem der "hybride Charakter" des Handwerks (Piegeler/Spars 2019: 14), der auf kundenspezifische Problemlösungen abzielt, legt Bezüge zum Value-Shop-Ansatz nahe.

Die Hybridität als „Bündelung von Industrieprodukten und Dienstleistung" (Kempermann/Lichtblau 2012: 2) und der Fokus auf kundenspezifisches Problemlösen lassen sich unter anderem auf die mit der Digitalisierung zunehmende technologische Komplexität zurückführen. So erfordert der Einsatz technologischer Innovationen meist den Erwerb zusätzlicher nachgelagerter Dienstleistungen (Piegeler/Spars 2019: 14). Hierzu passt die Debatte um eine zunehmende territoriale Servitization - also eine so genannte Verdienstleistung - des produzierenden Gewerbes (Lafuente/Vaillant/Vendrell-Herrero 2019: 313). Territoriale Servitization beschreibt - analog zu hybriden Geschäftsmodellen - die veränderte Integration von Dienstleistung und Produktion hin zu einer lokalen hybriden Wertschöpfungskette (De Propris/ Storai 2019: 390). Dazu wird die „spatial convergence of manufacturing and service firms [...] towards a symbiotic recoupling with a bounded spatial dimension" hervorgehoben, die auf erneute Agglomeration und Zentralisierungstendenzen der Produktion hinweist (De Propris/ Storai 2019: 391).

1 Unter Maker Movement werden Produktionsformen des ,Selbstmachens' verstanden, die tendenziell Produktion und Konsum vereinen und digitale Produktionstechnologien nutzen.
Zusammenfassend ist festzuhalten, dass bisherige Forschungen auf den relativen Bedeutungszuwachs städtischer Standorte für produzierende Unternehmen hindeuten, erstens aufgrund innovationssystemischer Qualitäten des Stadtraums, zweitens aufgrund seiner spezifischen Ressourcenausstattung und Fachkräfteverfügbarkeit. Zugleich könnte sich als Konsequenz des technologischen Wandels - das heißt auch von Industrie 4.0 - prinzipiell auch eine zunehmende symbiotische (Wieder)ankopplung von Produktion und Dienstleistung hin zu einer hybriden digitalen urbanen Produktion ergeben. Die Wirkungszusammenhänge, die diese erst in Anfängen erkennbaren Entwicklungstrends prägen, sind bislang jedoch unzureichend erforscht und von definitorischer Unschärfe geprägt. Noch fehlen Erkenntnisse darüber, ob sich bestehende urbane Produktionsunternehmen überhaupt in Richtung dieser digitalen urbanen Produktion verändern oder - was dieser Beitrag exploriert - inwiefern neue hybride Formen der Produktion im urbanen Raum entstehen.

\section{Konzeptionelle Rahmung und Typologie einer hybriden digitalen urbanen Produktion}

Als konzeptionelles Gerüst wird nachfolgend eine Typologie neuer Formen der hybriden digitalen urbanen Produktion vorgeschlagen. Auch wir gehen hierbei vom Trend in Richtung einer territorialen Servitization aus (De Propris/Storai 2019: 391). Dabei ist die Ko-Lokation von Produktions- und Vorproduktionsaktivität, wie beispielsweise Design sowie Forschung und Entwicklung (F\&E), hoch bedeutsam. Unsere Konzeptualisierung zum technologischen Wandel greift auf einen Ansatz zurück, der diese Ko-Lokation bereits kategorisiert (Pisano/Shih 2012b: $61 \mathrm{ff}$.) und bei wirtschaftsgeographischen Untersuchungen sowohl globale (Ivarsson/Alvstam/Vahlne 2017: 152; van Assche 2017: 745 ff.) als auch lokale Zusammenhänge (Buciuni/Pisano 2018: 1073) berücksichtigt hat. Die Ko-Lokation von Vorproduktion und Produktion wird dabei als abhängig vom technologischen Reifegrad der angewandten Produktionsprozesse sowie von der Modularität der hergestellten Produkte erachtet (Pisano/ Shih 2012a: 97 ff.). Daraus ergeben sich vier Kombinationen, die in Abbildung 1 dargestellt sind. Auf diese Weise lässt sich die hybride digitale urbane Produktion als Verbindung von Produktions- und Konzeptionstätigkeiten und deren Standortanforderungen erfassen. 


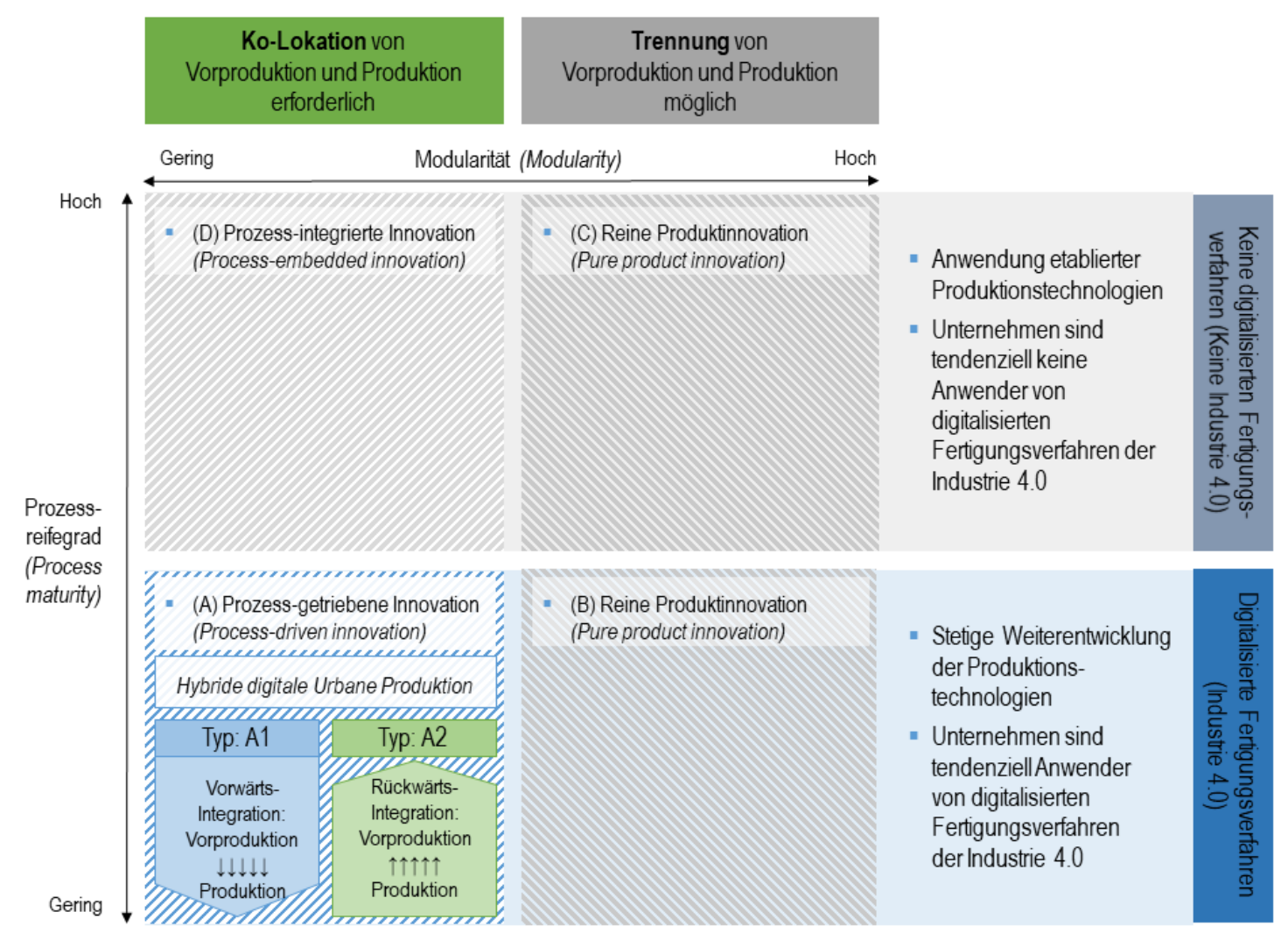

Abbildung 1: Hybride digitale urbane Produktion in der Modularität-Reifegrad-Matrix Quelle: Eigene Darstellung in Anlehnung an Pisano/Shih (2012a: 98) und Pisano/Shih (2012b: 66)

Unter dem Prozessreifegrad verstehen Pisano und Shih (2012a: 97) vor allem "how much a process has evolved rather than the age of a technology, although obviously the two tend to be correlated". Unterscheidet man hierbei einen geringen und einen hohen Reifegrad, so können die digitalisierten Prozesstechnologien innerhalb der Industrie 4.0 als Technologien mit noch nicht voll ausgeschöpftem Entwicklungspotenzial gelten; sie weisen somit einen relativ geringen Prozessreifegrad auf. Denn meist werden diese Technologien (derzeit noch) als Prototypen oder Modellversuche angewandt. Die zweite Dimension Modularität bezieht sich auf die Interdependenz von Produktion und Vorproduktion (das heißt Design bzw. F\&E). Ein hoher Grad an Modularität, also eine relativ geringe Interdependenz von Konzeption und Produktion, erfordert einen hohen Anteil kodifizierten Wissens, erlaubt aber tendenziell eine räumliche Trennung dieser Funktionen (Typen B und C in Abbildung 1). Ein geringer Grad an Modularität, das heißt eine relativ hohe Interdependenz von Konzeptions- und Produktionstätigkeiten, verlangt dagegen ein hohes Maß an impliziertem Wissen (tacit knowledge) (Gertler 2003: 76) und somit die räumliche Ko-Lokation, da sonst höhere Transaktionskosten drohen (van Assche 2017: 747) (Typen A und D in Abbildung 1).

Im weiteren Verlauf betrachten wir die Typen $A$ und $B$, denn nur hier ist die Anwendung von Industrie4.0-Technologien relevant. Diese Typen kombinieren einen geringen Prozessreifegrad mit geringer (A) bzw. hoher (B) Modularität. Bei Typ B gibt es zwar - aufgrund des geringen Prozessreifegrades - fortgesetzt neue Prozesserneuerungen, doch ist wegen der hohen Modularität nicht zwingend notwendig, Konzeption und Produktion am selben Standort zu vereinen. Der urbane Standort bringt somit erwartbar weniger Vorteile. Weil die hoch modularisierte Produktion meist mit der Produktion großer Stückzahlen einhergeht, rechtfertigt nur eine entsprechend hohe Nachfrage die betriebswirtschaftliche Entscheidung der Standardisierung der Produktionsprozesse. Für hybride digitale urbane Produktion erscheint die großserielle Massenfertigung aufgrund verschiedener stadtraumspezifischer Standortnachteile dagegen weniger vorteilhaft (vgl. auch Stiehm 2017: 64 f.). 
Anders verhält es sich bei Typ A, bei dem Änderungen des Produktionsprozesses drastische Produktänderungen hervorrufen können (Pisano/Shih 2012a: 98). Die Interdependenz von Konzeption und Produktion ist bei diesem wenig modularisierten Typ besonders hoch, was räumliche Ko-Lokation verlangt. Hier werden eher Kleinserien oder Einzelanfertigungen produziert, die einen hohen Individualisierungsgrad aufweisen (vgl. Stiehm 2017: 64 f.). Für Typ A sind daher höhere Urbanitätsvorteile zu erwarten und es kann von einer strategischen Präferenz für städtische Standorte ausgegangen werden. Daher bildet Typ A hybride digitale urbane Produktion am ehesten ab, wir verorten sie demnach im unteren linken Quadranten von Abbildung 1.

Typ A - als Konversion verschiedener Stufen der Wertschöpfung hin zu einer hybriden Produktion - lässt sich in weitere Analyseeinheiten unterteilen, gemäß der möglichen Integrationsrichtung in der Wertschöpfungskette. Ein Betrieb kann prinzipiell von Konzeptions-Tätigkeiten ausgehend Teile der Produktion vorwärts mit einbinden (Typ A1) oder von der Produktion ausgehend Teile der Konzeption rückwärts integrieren (Typ A2). Beispiele sind etwa Ingenieurdienstleister für Typ A1 und produzierende Handwerksunternehmen für Typ A2. Beide Typen sind erwartbar mit unterschiedlichen Auswirkungen auf die betrieblichen Geschäftsfelder verbunden: Vorwärts integrierende Unternehmen (A1) tendieren dazu, Produktinnovationen hervorzubringen, während rückwärts integrierende Unternehmen (A2) eher neue Abschnitte der Wertschöpfungskette besetzen und Prozessinnovationen kreieren. Die Typen A1 und A2 sind in Abbildung 1 als Unterkategorien des Typs A in vertikal vorwärts bzw. rückwärts orientierter Integrationsrichtung schematisch dargestellt. Dieser Konzeptrahmen soll das Wirkungsgefüge der innerbetrieblichen Wertschöpfungsprozesse im Zuge der digitalen urbanen Produktion und folglich auch deren spezifische Standorteinbettung erklären helfen, wie im nachfolgend vorgestellten empirischen Ansatz eingehender untersucht.

\section{Untersuchungsgebiet und Forschungsmethodik}

Wie der technologische Wandel bzw. die aufkommende Industrie 4.0 die urban verortete Produktion verändern könnte, wurde vor allem gestützt auf eine Primärdatengewinnung gemäß eines explorativ-qualitativen Forschungsdesigns untersucht, neben umfassenden Sekundärdatenanalysen (vgl. Fuchs/Fromhold-Eisebith/
Busch et al. 2017: 26 ff.). Die empirischen Arbeiten konzentrieren sich auf das Bundesland Nordrhein-Westfalen, welches stark vom produzierenden Gewerbe geprägt ist, auf eine lange Industrietradition zurückblickt (Birch/ MacKinnon/Cumbers 2010: 39) und zudem wachsende Verflechtungen zwischen der industriellen Basis und regionalen Dienstleistungen aufweist (Hamm 2013: 97). Innerhalb Nordrhein-Westfalens wurden acht Stadtregionen für die Untersuchung ausgewählt: Aachen, Dortmund, Duisburg, Düsseldorf, Köln sowie Remscheid, Solingen und Wuppertal. Die ausgewählten Stadtregionen weisen einerseits die Gemeinsamkeit eines ausgeprägten industriellen Erbes auf. So stehen alle acht Stadtregionen vor besonderen Herausforderungen der Nutzung neuer digitaler Produktionstechnologien im Sinne von Industrie 4.0. Sie zeichnen sich andererseits durch ihre explizite Verschiedenheit aus: Ortsspezifische infrastrukturelle und institutionelle Rahmenbedingungen, Branchen- und Betriebsstrukturen sowie unterschiedliche technologische Entwicklungspfade machen die Untersuchungsstädte jeweils zu Beispielen verschiedener emblematischer Raumtypen.

Zur Datengewinnung wurden im Zeitraum 2017 bis 2018 insgesamt 41 leitfadengestützte Experteninterviews mit verschiedenen Funktionsträgern geführt (Methodik nach Mattissek/Pfaffenbach/Reuber 2013: 189). Die erste Empiriephase umfasste Gespräche mit Vertreterinnen/Vertretern von Verbänden und anderen Organisationen, wie Gewerkschaften, Handwerkskammern, Industrie- und Handelskammern, Wirtschaftsförderungsgesellschaften sowie dem Verein Deutscher Ingenieure. In der zweiten Empiriephase (2018) kamen 17 Gespräche mit Vertreterinnen/Vertretern aus urban produzierenden Unternehmen hinzu, die in 15 Fällen vor Ort geführt wurden (teils mit Betriebsbesichtigung). Diese Unternehmen bezeichnen sich selbst als Anwender digitalisierter Fertigungsverfahren der Industrie 4.0 und wurden durch Referenzen der Befragten in Phase 1 identifiziert. Die Analyse der Interviewdaten erfolgte in Anlehnung an Mayring (2000: 4).

Die Ergebnisse der interregional vergleichenden Ansätze im Forschungsprojekt, die beispielsweise auch stadtspezifische Akteurkonstellationen und Branchendynamiken beleuchten, wurden bereits an anderer Stelle publiziert (vgl. Fuchs/Fromhold-Eisebith/Busch et al. 2017: 26 ff.; Mühl/Busch/Fromhold-Eisebith et al. 2019: $60 \mathrm{ff}$.). Aufbauend auf diesen Arbeiten legt der vorliegende Beitrag vertiefte empirische Erkenntnisse zu Fallbeispielen der in Kapitel 3 definierten Idealtypen hybrider digitaler urbaner Produktion dar (A1 und A2). Die vorgestellten Beispiele stehen stellvertretend für die von 
uns übergreifend untersuchte neue Kategorie: Es sind produzierende Handwerksunternehmen und Ingenieurdienstleister, die am urbanen Standort digitale Produktionstechnologien einsetzen (von insgesamt 17 in die Studie einbezogenen Unternehmen waren sechs Ingenieurdienstleister und vier Handwerksbetriebe), wobei sich alle zehn als hybride digitale urbane Produzenten den oben genannten Typen zuordnen lassen. Dies vermag somit bereits eine gewisse empirische Relevanz dieser neuen hybriden Typen nachzuweisen; wenngleich auch quantitative Erfassungen der Fallzahlen aufgrund der definitorischen Unschärfe schwerfallen.

\section{Empirische Ergebnisse und Analyse: Fallbeispiele digitalisierter urbaner Produktion in Nordrhein-Westfalen}

Nachfolgend wird aufgezeigt, inwiefern durch digitale Fertigungsverfahren der Industrie 4.0 neue Formen einer hybriden digitalen Produktion im urbanen Raum aufkommen. Interessant ist dabei vor allem, wie die Technologien der Industrie 4.0 neue Verbindungen von industrieller und handwerklicher Produktion im Stadtraum entstehen lassen. Unser Konzeptrahmen (Kapitel 3) legt nahe, dass ein urbaner Standort für Unternehmen mit bestimmten Merkmalen besonders vorteilhaft sein könnte, konkret für die oben als Typ A1 und A2 gekennzeichneten Varianten hybrider digitaler urbaner Produktion. Für diese beiden Typen bietet der Stadtraum vielfältige Urbanisationsvorteile. Diese Produzenten betreiben vor allem eine gewerbliche Nutzung von 3D-Druck und anderen Technologien, die Konstruktion und Produktion verbinden. Kleine Industrieunternehmen und Handwerksbetriebe realisieren damit hybride Geschäftsmodelle an der Schnittstelle zwischen Ingenieurdienstleistung und handwerklich-industrieller Produktion.

Die aufkommenden digitalen Produktionstechnologien lassen Produktion und Konstruktion am selben Standort zunehmend verschmelzen. Abbildung 2 zeigt schematisch die Wertschöpfungsprozesse zweier Unternehmenstypen, die weiter unten eingehender vorgestellt werden: digital produzierende Ingenieurdienstleister (Abbildung 2, links) sowie digital produzierende Handwerksunternehmen (Abbildung 2, rechts). Die Wertschöpfungsprozesse sind zur Illustration zunächst als sequenzielle Ketten abgebildet. Abbildung 2 links zeigt modellhaft den Arbeitsprozess der Ingenieurdienstleister, deren Hauptgeschäftsfelder im Bereich ,klassischer" Ingenieurstätigkeiten liegen, wie Konzeption, Konstruktion und Arbeit mit CAD-Programmen. Diese Bereiche werden nun, ermöglicht durch digitale, flächenschonende Fertigungsverfahren, um weitere Tätigkeiten ergänzt; es findet eine vertikale Integration nachgelagerter Stufen der Wertschöpfungskette statt. Die Fallbeispiele dieses Typs A1 zeigen, dass solche Ingenieurdienstleister nun zunehmend in der Lage sind, Prototypen sowie kleinserielle Produkte zu fertigen (vgl. Kapitel 5.1). Dies reicht bis zur Produktion und Vermarktung eigener Designs. Ein Ingenieurdienstleister wird also zum digitalen ,Ingenieurproduzenten'. Dies ermöglicht zusätzlich auch Produktinnovationen.

Im Gegenzug werden Handwerksunternehmen (Abbildung 2, rechts) nun von Handwerksproduzenten zu ,Handwerksingenieuren'. Neben der Hauptaktivität der ,klassischen' Fertigung von Produkten in Handarbeit integrieren Handwerksunternehmen vertikal vorgelagerte Bereiche der Wertschöpfungskette. Zunehmend führen sie somit auch konzeptionelle Tätigkeiten der Konstruktion (z. B. mittels CAD-Systemen) durch und erschließen auf diese Weise weitere Geschäftsfelder. Die Fallbeispiele des Typs A2 zeigen, dass der Computer Teil des handwerklichen Werkzeugkastens geworden ist (vgl. Kapitel 5.2). Die Grenzen zwischen beiden Entwicklungsvarianten sind allerdings fließend und daher lassen sich diese neuen Formen der digitalen urbanen Produktion auch als hybride Geschäftsmodelle an der Schnittstelle zwischen Ingenieurdienstleistung (Typ A1) und Handwerksproduktion (Typ A2) bezeichnen.

Die nachfolgend vertieften Fallbeispiele stellen anschaulich diese neuen Typen dar. Sie demonstrieren und illustrieren - stellvertretend für alle von uns empirisch erfassten Phänomene - die hybride Verschmelzung von kognitiv-konzeptionellen Tätigkeiten in Konstruktion und Design involvierter Ingenieurbüros einerseits und handwerklich-kreativen Tätigkeiten auf materielle Endprodukte spezialisierter Handwerksbetriebe andererseits. Diese hybride Verschmelzung erläutern wir zunächst für Ingenieurdienstleister (A1) und dann für Handwerksunternehmen (A2). Die Vorstellung der Beispiele für die Typen A1 und A2 nutzt zur Charakterisierung die Aspekte (1) Technologieeinsatz, (2) Individualisierung und (3) Standorteinbettung. Als Querschnittsdimension betrachten wir bezogen auf alle drei Aspekte zudem die Wertschöpfungsprozesse. 


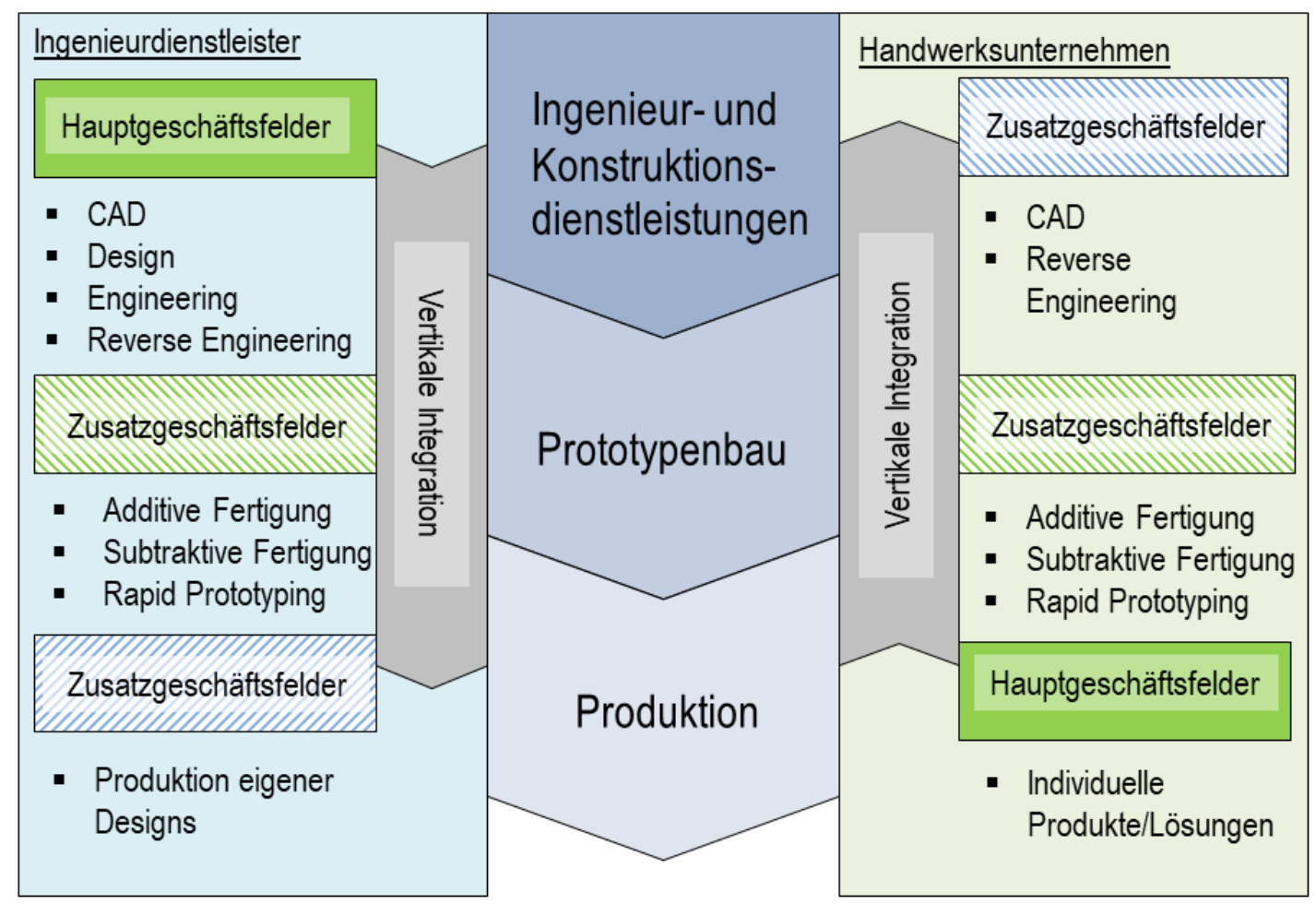

Abbildung 2: Vertikale Integration entlang des Wertschöpfungsprozesses digitaler urbaner Produktion

\subsection{Fallbeispiele zum Typ A1: „Digitale urbane Ingenieurproduzenten"}

\subsubsection{Technologieeinsatz}

Nachfolgend werden verschiedene Fallbeispiele hybrider digitaler urbaner Produzenten im Bereich der Ingenieurdienstleister vorgestellt. Diese Unternehmen erstellen neben der Konzeption neuer Produkte unter Anwendung digitaler Produktionstechnologien auch Prototypen bis hin zu ersten Musterserien, vorwiegend als Zulieferer für Geschäftskunden (Business-to-Business-Märkte (B2B)). So können diese prototypenhaften Produkte dann bereits am Markt durch die Kunden getestet werden. Ein Duisburger Ingenieurdienstleister artikuliert dies wie folgt: „Wir haben durch das Zusammenspiel verschiedener Technologiefelder, wie Laser-cut, 3D-Scanning, Reverse Engineering und 3D-Druck, ganz andere Möglichkeiten, den Unternehmen Produkte aus einer Hand anzubieten. Wenn ich Anbauteile für Autos realisiere, können wir [nicht nur] direkt die Karosserie einscannen, dann digital die Produkte mit dem Kunden zusammen entwickeln und über 3D-Druck nicht nur Prototypen ausgeben, sondern [auch] erste Musterserien [anfertigen], die unsere Kunden auch direkt verkaufen können. Das heißt, sie können einschätzen, wie der Markt auf die Produkte reagiert."

Der Duisburger Ingenieurdienstleister erwartet, dass sich zunehmend auch Produktionsschritte mit digitalen Verfahren, wie dem 3D-Druck, verschneiden werden, was mittelfristig zu einer flexibleren Produktion führen kann. Hier ändern sich entsprechend auch die Anforderungen an die Qualifikationen der Mitarbeiterinnen und Mitarbeiter, sodass eine Kombination aus handwerklichpraktischen Fähigkeiten sowie ingenieurwissenschaftlichen Kenntnissen benötigt wird. Den hohen Stellenwert der IT-Kenntnisse bei den Mitarbeiterinnen und Mitarbeitern artikuliert ein weiterer Unternehmer aus Dortmund: „Es ergibt Sinn, dass unsere Mitarbeiter mehr handwerkliches Geschick haben, [...] aber alles, was wir machen, ist immer digital, und wir könnten niemanden gebrauchen, der nicht mit CAD umgehen kann. Das ist eine Grundvoraussetzung, weil bei uns eigentlich alles digital abläuft."

\subsubsection{Individualisierung}

Es zeigt sich, dass diese ,Ingenieurproduktion' hauptsächlich in kleinen Serien erfolgt und für größer skalierte 
Bestellungen Verträge mit Auftragsfertigern geschlossen werden, denn die eigenen Produktionskapazitäten der Ingenieurdienstleister reichen für eine Massenproduktion in der Regel nicht aus. Dies schildert ein Düsseldorfer Ingenieurdienstleister im Bereich spezifischer loT ${ }^{2}$-Produkte: „Wir haben hier im Haus eine kleine Produktion, mit der wir zwei bis fünf Platinen herstellen. Oder teilweise bis zu 25 [Platinen] am Stück. Das dauert etwas länger, weil es eine halbautomatische Arbeit ist; wir haben einen halbautomatisierten Roboter. Dann haben wir eine Produktionsstraße, die ist im Moment im Aufbau hier [in Düsseldorf]. Dort haben wir vier Maschinen und dort werden wir dann alles bestücken, was an die 100 Stück geht. Sobald wir aber für den Kunden 1.000 oder 10.000 herstellen und die [Produkte] verpacken müssen, haben wir dann einen Auftragsfertiger in Mönchengladbach, der diesen Prozess komplett begleitet."

Oft sind die Ingenieurdienstleister auch durch die produktionstechnischen Möglichkeiten eingeschränkt. Ihnen steht nur ein begrenztes Instrumentarium zur Verfügung, welches zwar im Ideenfindungs- und Konzeptionsprozess ausreicht, jedoch nicht zur groß skalierten Produktion genügt. So wird für die nächste Skalierungsstufe auf industrielle Partner zurückgegriffen, die über das notwendige produktionstechnische Know-how sowie eine für großserielle Produktion geeignete Infrastruktur verfügen. Dies schildert der Dortmunder Ingenieurdienstleister folgendermaßen: „Wir haben eine kleine Werkstatt: da stehen zwei 3D-Drucker, eine Bohrmaschine, eine Säge und es gibt noch zwei PCs, auf denen werden die CAD-Zeichnungen angefertigt. [...] Aber das ist natürlich ein ganz kleines Spektrum, was wir da abbilden können. Da geht uns dann sehr schnell die Luft aus und dann müssen wir auf unser Netzwerk zurückgreifen."

Eine ähnliche Sicht auf die hybride digitale urbane Produktion zeigt sich auch bei öffentlichen Meinungsträgern: Die Duisburger Wirtschaftsförderung artikuliert beispielsweise, dass der urbane Raum für die größer skalierte Produktion potenziell weniger Vorteile bietet, sodass ein Standort im außerstädtischen Bereich präferiert würde: „Unternehmen [haben] aufgrund ihrer [...] geringen Produktionsgrößen überhaupt erst die Möglichkeit innerhalb einer Stadt [...] zu produzieren. Das machen sie dann nicht, weil sie einen 3D-Drucker haben, sondern sie nehmen den 3D-Drucker, weil sie damit kleine Stückzahlen produzieren wollen. [...] Sobald aber das gleiche Unternehmen auf einmal vor dem Problem steht, anstelle von 20 Produkten im Monat 20.000 [Pro-

2 Internet of Things dukte] auszuliefern, muss es sich entsprechende Produktionsräume besorgen. Und die findet es nicht im urbanen Bereich." Dies zeigt, dass nicht allein die Lage im innerstädtischen Bereich relevant ist, sondern eher die unmittelbare Nähe zu Kundinnen/Kunden und Partnerinnen/Partnern ausschlaggebend ist. Zwar erfolgen solche Kontakte oft innerhalb von Stadtregionen. In einem polyzentrischen System wie in Deutschland liegen diese Knotenpunkte aber auch abseits der großen Metropolregionen mit ihren ausgeprägten kreativen Milieus. Oft sind es Klein- und Mittelstädte, in denen Headquarters großer Unternehmen und mittelständischer Hidden Champions angesiedelt sind.

\subsubsection{Standorteinbettung}

Die digitalen Ingenieurdienstleister profitieren ausdrücklich vom urbanen Umfeld, dabei vor allem von der Nähe zu Fachkräften, der digitalen Infrastruktur sowie urbanen Netzwerken. Der Dortmunder Unternehmer verdeutlicht dies: „Ich brauche natürlich meine Programmierer und gute Leute, die sich mit 3D-Druckverfahren auskennen. Und wenn mein Standort 80 Kilometer von der Stadt entfernt ist, dann werde ich die nicht bekommen, also da ist eine Absage dann garantiert." Ähnlich äußert sich auch der Geschäftsführer eines Kölner Digitalunternehmens: „Man muss diese Nähe haben, denn so ein ,Kreativling“ ist so knapp. Wenn du inn findest, sagt er: Ich habe keine Lust, nach Marsdorf [Vorort von Köln] zu fahren. Entweder arbeite ich von zu Hause oder du hast ein ordentliches, schönes Büro in Köln."

Die Nähe dieser Unternehmen zu Kundinnen und Kunden im urbanen Raum ermöglicht außerdem kürzere Reaktionszeiten auf geänderte Anforderungen und einen vereinfachten Informationsaustausch. Dies unterstreicht die nach wie vor hohe Wichtigkeit von Face-to-faceKontakten. Nachfolgend wird dies durch den Duisburger Unternehmer veranschaulicht: „Wir haben gemerkt: Je näher man bei seinen Kunden sitzt, umso einfacher ist es, Projekte umzusetzen. [...] Bei aller Liebe zu neuen digitalen Technologien, der räumliche Kontakt kann durch nichts ersetzt werden. Man kann sicherlich durch Skype viel ersetzen oder viel vereinfachen. [...] Bis auf den Erstkontakt beim Kunden oder ein Auftragsgespräch zum Projekt läuft bei uns auch alles digital ab: Wir arbeiten bei uns hier im Büro, senden Daten über E-Mail oder FTP-Server raus. Das heißt, wir müssen dafür nicht mehr beim Kunden sein, aber um ein Projekt zu besprechen, ist es immer hilfreicher, wenn man beim Kunden direkt vor Ort ist. Das schätzen die Kunden auch, weil auch nicht 
jeder immer Lust auf eine Skype-Telefon-Konferenz hat. Die Kunden wollen einfach, dass man bei innen vor Ort ist. Das war auch der Grund, warum wir früher, als noch die meisten unserer Kunden quer aus ganz Deutschland kamen, immer häufiger Projektschwierigkeiten hatten; weil wir eben nicht mal schnell vor Ort sein konnten." Die Konstellation, in der diese Nähe eine große Rolle spielt, wurde insbesondere zwischen großen Unternehmen und den beschriebenen kleinen und mittleren Ingenieurunternehmen beobachtet. Denn Ingenieurdienstleister können nicht nur in Innovations- und Kreationsprozessen flexibler reagieren, sondern sind - aufgrund ihrer Größe - meist auch weniger stark reguliert als große Unternehmen und operieren somit gegebenenfalls zu günstigeren Lohnkosten.

\subsection{Fallbeispiele zum Typ A2: „Digitale urbane Handwerksingenieure“"}

\subsubsection{Technologieeinsatz}

Auch bei den nachfolgend dargelegten, typischen Fallbeispielen digital urban produzierender Handwerksunternehmen gehört der Kundenstamm vorwiegend zum Geschäftskundensegment. Es werden anspruchsvolle, teils technisch komplizierte Anforderungen an diese Betriebe gestellt. Dabei zeigt sich, dass hier die gleichen bzw. ähnliche Technologien zum Einsatz kommen wie bei den Ingenieurdienstleistern (Typ A1). Ähnlich sind auch die Aufgabenstellungen, wie beispielsweise das Anfertigen von Prototypen oder Kleinserien. Dies wird vom Geschäftsführer einer Wuppertaler Schreinerei wie folgt dargelegt: „Wir fertigen keine eigenen Produkte und verkaufen diese am Markt, sondern werden von einem Kunden gefragt, ob wir ein Produkt fertigen können. Das sind immer sehr außergewöhnliche Sachen, die man in der normalen Schreinerei nicht bekommt. Wir haben hier zum Beispiel einen Prototyp, für den eine Form anzufertigen war. Eine einmalige Form aus einem ganz speziellen Material, und dieses Material reproduzieren wir dann in Holz, sodass man dann ein ganz individuelles Produkt hat. Das sind halt Sachen, die wird eine normale Schreinerei nicht umsetzen können, und wenn, dann wahrscheinlich auf sehr komplizierten Wegen. Bei uns läuft das einfach dadurch, dass wir auch über einen [3D-]Scanner verfügen. So können wir solche Formen einscannen und dann reproduzieren."

Die Lösungen werden somit durch die Kombination ,klassischer' handwerklicher Fertigkeiten mit digitalen Produktionstechnologien konzipiert und umgesetzt.
Jeder Auftrag zeichnet sich durch hohe Individualität aus und erfordert flexible Problemlösungskompetenzen. Der Geschäftsführer eines Kölner Handwerksunternehmens schildert dies folgendermaßen: „Das ,richtige Handwerk verschmilzt sehr mit den ingenieurlastigen Berufen, weil man auch sehr viel in die Konstruktion geht." Der betriebliche "Werkzeugkasten" wird, ausgehend von herkömmlichen handwerklichen Produktionsweisen, de facto zunehmend um digitale Prozessschritte erweitert (z. B. CAD-Systeme in Kombination mit 3D-Druck oder Mehr-Achs-CNC-Maschinen). Die Handwerksbetriebe nehmen zusätzliche (digitale) Komponenten in ihr Leistungsspektrum auf, integrieren vorgelagerte Abschnitte in ihre Wertschöpfungskette. Ein Handwerksunternehmer aus Remscheid plädiert dennoch dafür, auch beim wachsenden Einsatz digitaler Produktionstechnologien die handwerklichen Kernkompetenzen weiterhin zu betonen: „Was man gerade bei dem Thema Urbane Produktion nicht aus den Augen verlieren sollte: Digitalisierung ist wichtig [...]. Aber man sollte die Vergangenheit oder das Klassische nicht vergessen oder verdrängen. Weil irgendwo daraus ergibt sich die Schnittmenge, die für den Kunden das optimale Portfolio bildet."

Der Geschäftsführer eines Kölner Handwerksunternehmens bringt die resultierende Verschmelzung von konzeptionellen Tätigkeiten der Konstruktion, die vormals spezialisierten Ingenieurdienstleistern vorbehalten war, mit der handwerklichen Produktion im Interview auf den Punkt. In dieser Schnittmenge der Kompetenzen sieht er eine besondere Marktnische, welche die Industrie in großserieller Fertigung nicht profitabel abdecken kann: „Die Industrie sagt meist: Wir machen die Konstruktion oder die Fertigung. [...] Bei uns [im Handwerk] merkt man bei den Projekten, dass das eine ohne das andere nicht geht. Also muss man entweder Erfahrung haben oder einfach sagen, dass es so oder so sein könnte. Dann melde ich mich in zwei Stunden nochmal. Dann geht es für zwei Stunden in die Werkstatt, wir bauen das Produkt grob zur Probe, um zu sehen, was funktioniert. Dann können wir schneller agieren." Das Zitat verdeutlicht neben der vertikalen Integration wichtiger Prozessschritte auch den iterativen und zirkulären Problemlösungskreislauf, der konzeptionelle und handwerklich-praktische Tätigkeiten miteinander verknüpft. Digitale Produktionstechnologien, wie CAD in Kombination mit CNC- oder 3D-Druckverfahren, wirken hier als Scharnier zwischen konzeptionellen und produktionsbezogenen Aufgaben. 


\subsubsection{Individualisierung}

Noch weitere industriell-handwerklich tätige Unternehmen sehen diese Scharnierfunktion als komparativen Vorteil, wie ein Beispiel aus Remscheid zeigt. Denn so können hoch individuelle Lösungen für Kundenkreise jenseits der groß skalierten Industriefertigung angeboten werden, die aber dennoch eine potenzielle Replizierbarkeit durch die digitale Speicherung der CAD-Dateien ermöglichen, wie der Geschäftsführer des Unternehmens schildert: „Ich sehe das Ganze hier als Verknüpfung zwischen klassischem Handwerk und der Digitalisierung. Warum? Natürlich arbeite ich mit 2D- und 3D-Konstruktionen. Das heißt, ich habe meine Bauteile alle digital auf dem PC vorhanden. Egal, ob es ein Werkzeug ist, ob es eine Maschinenbauplatte ist oder ob es ein Ersatzteil für einen Landwirt ist. [...] Ich kann die Sachen - was für mich auch schon zur Digitalisierung gehört - erstmal als Prototyp im 3D-Druckverfahren drucken. Das ist für mich Digitalisierung."

\subsubsection{Standorteinbettung}

Ein in Köln besuchtes Handwerksunternehmen hat einen innerstädtischen Standort in direkter Nachbarschaft zu Wohnbebauung sowie weiteren Handwerksund Produktionsbetrieben. Obwohl die Kundennähe hier teils als vorteilhaft bewertet wird, nimmt der Interviewpartner vor allem die Vorteile der urbanen Lage für die eigenen Mitarbeiter wahr: „[Die städtische Lage] ist tatsächlich etwas für unsere Mitarbeiter, die können mit dem Fahrrad hier hinfahren, das ist super. Dafür ist das klasse." Darüber hinaus entstehen durch die Nähe des Unternehmens zu den Wohnorten der Mitarbeiterinnen und Mitarbeiter weitere produktivitätsbezogene Synergien: „Dass die Mitarbeiter in der Nähe sind und dass sie zwar nur bis fünf Uhr arbeiten, aber gerne bis $20 \mathrm{Uhr}$ hier sitzen und vielleicht noch etwas kochen [ist ein Vorteil]. Und wenn die CNC-Fräse läuft, [...] dann nehmen wir auch abends eine Platte runter und legen das nächste Stück drauf und bestücken die Maschine für die Nacht."

Im Gesamtbild scheint der Einsatz neuer digitaler Produktionstechnologien prinzipiell eine Konvergenz der kognitiv-konzeptionellen Tätigkeiten des Ingenieurdienstleisters und der handwerklich-kreativen Tätigkeiten des Handwerksbetriebes voranzutreiben. Beide Geschäftsmodelle weisen Gemeinsamkeiten einer Marktorientierung in Richtung spezialisierter Nischenlösungen auf, oftmals für Geschäftskunden (B2B-Märkte). Dies zieht auch weitere Implikationen der Standortwahl nach sich, wie ein Aachener Kreativ-Unternehmer verdeutlicht: „Für Privatkunden wäre der Fokus, im urbanen Raum zu sein, nett, aber nicht zwingend eine Voraussetzung. Wenn wir das aber für Geschäftskunden betreiben, ist es sicherlich wichtig, vor Ort zu sein, weil das Absprechen von Aufträgen und der Zeitdruck, um das Ganze erledigen zu können, sehr wichtig sind. Das bekommen wir immer wieder von unseren Kunden gespiegelt, dass es sehr geschätzt wird, wenn die Wege kurz sind."

Neben den oben vorgestellten Fällen haben unsere empirischen Arbeiten weitere Beispiele für Betriebe der Bereiche IoT-Anwendungen, 3D-Scan-Anwendungen, Herstellung komplexer Maschinenbauteile oder spezieller Elektronikelemente erfasst. Auch sie sind durch zirkulär-iterative Problemlösungsansätze mit Scharnierfunktion digitaler Produktionstechnologien geprägt: Digitale Konzeption und Konstruktion am Computer und physisch-handwerkliche Produktion mittels digitaler Produktionstechnologien wechseln sich in den Iterationen so lange $a b$, bis der (Kunden)auftrag erfüllt ist. Die Empirie bestätigt, dass sich die Typen A1 und A2 lediglich hinsichtlich der Ausrichtung der vertikalen Integration in der Wertschöpfungskette unterscheiden: Digitale Produktionstechnologien erlauben, einerseits das Tätigkeitsspektrum des, Ingenieurproduzenten “ - vorwärts integrierend - um Produktion und andererseits das Tätigkeitsspektrum des ,Handwerksingenieurs' - rückwärts integrierend - um Konstruktion zu erweitern. Diese Trends können als erwiesene Kennzeichen für neue Formen hybrider digitaler urbaner Produktion gelten.

\section{Diskussion der Ergebnisse: Wertschöpfung und standörtliche Einbettung hybrider digitaler urbaner Produktion}

Wie aufgezeigt, entwickeln sich sowohl Ingenieurdienstleister als auch Handwerksunternehmen unter Einsatz digitaler Produktionstechnologien in Richtung einer hybriden digitalen urbanen Produktion. Während sich die Typen A1 und A2 bei der Integrationsrichtung im Wertschöpfungsprozess unterscheiden, besteht jedoch relativ wenig Differenzierung in Bezug auf die eingesetzten Produktionstechnologien. Einigende Merkmale der hybriden digitalen urbanen Produktion sind außerdem der Fokus auf kundenspezifische Individuallösungen und ein verstärkter Einbezug der Kundinnen und Kunden, 
insbesondere durch digitale Kommunikationskanäle. Die aus den Fallbeispielen gezogenen Erkenntnisse zu einigen generellen Kennzeichen der hybriden digitalen urbanen Produktion sollen nun zielführend mit den theoretischen Überlegungen aus den Kapiteln 2 und 3 in Verbindung gebracht werden, um weiterführende konzeptionelle Erkenntnisse abzuleiten. Besonderes Augenmerk legen wir auf die Wertschöpfungsaktivität der Unternehmen und deren Einbettung in den urbanen Standort. Zur Konzeption des Wertschöpfungsprozesses bei „offenen Werkstätten" im urbanen Raum (Lange/Bürkner 2018b: 683) wurde bereits in Kapitel 2 der Value-Shop-Ansatz eingeführt (Stabell/Fjeldstad 1998: 420; Lange/Bürkner 2018a: 99).

Die bei unserer Untersuchung identifizierten urbanen ,Ingenieurproduzenten“ bzw. ,Handwerksingenieure', die sowohl wissensintensive Dienstleistungen als auch iterative Werkstattprozesse zu bieten haben, lassen sich aufgrund des zirkulären Problemlösungscharakters gut mit dem Value-Shop-Ansatz verbinden, ähnlich wie dies schon bei sonstigen Analysen gewandelter Unternehmensformen erfolgt ist (z. B. Glückler 2007: 958; Lange/ Bürkner 2018a: 99). Als Basis für die konzeptionelle Weiterentwicklung stellt Abbildung 3 den Problemlösungszyklus der hybriden digitalen urbanen Produktion angelehnt an den Value-Shop-Ansatz dar: Nachdem mit Eingang des Kundenauftrags in Schritt (1) die Problemdefinition zur spezifischen technischen Aufgabenstellung erfolgt ist, sind die Schritte (2), (3) und (4) jeweils als Teil eines zirkulären Prozesses zu verstehen (mittlerer Ring in Abbildung 3): Zunächst werden Lösungsalternativen ermittelt (2), aus diesen wird dann - eventuell nach mehrmaligem Zyklus-Durchlauf - eine passende Lösung ausgewählt (3), um sie letztlich in der Produktion umzusetzen (4) (Stabell/Fjeldstad 1998: 424). Der resultierende Lösungsvorschlag wird dann noch evaluiert (5); der Zyklus endet, wenn jener Vorschlag das anfängliche Kundenproblem de facto löst.

Adaptiert man den Problemlösungszyklus bzw. Value-Shop-Kreislaufprozess für die digitale urbane Produktion, sollten unseren Untersuchungen zufolge zwei weitere interaktive Prozesse ergänzt werden. Erstens wird der Zyklus durch ein Kundenproblem initiiert, beispielsweise den Bedarf zur Anfertigung eines spezifischen Bauteils, was zur Auftragsvergabe an einen digitalen Ingenieurdienstleister oder Handwerksbetrieb führt. Es kommt zu kontinuierlichen Rückkopplungen mit der Kundin/dem Kunden (oberer Kreislauf in Abbildung 3), die letztlich erst bei der Annahme der finalen Lösung durch die Kundschaft enden. Die prominente Rolle und enge Einbindung solcher Kundenbeziehung

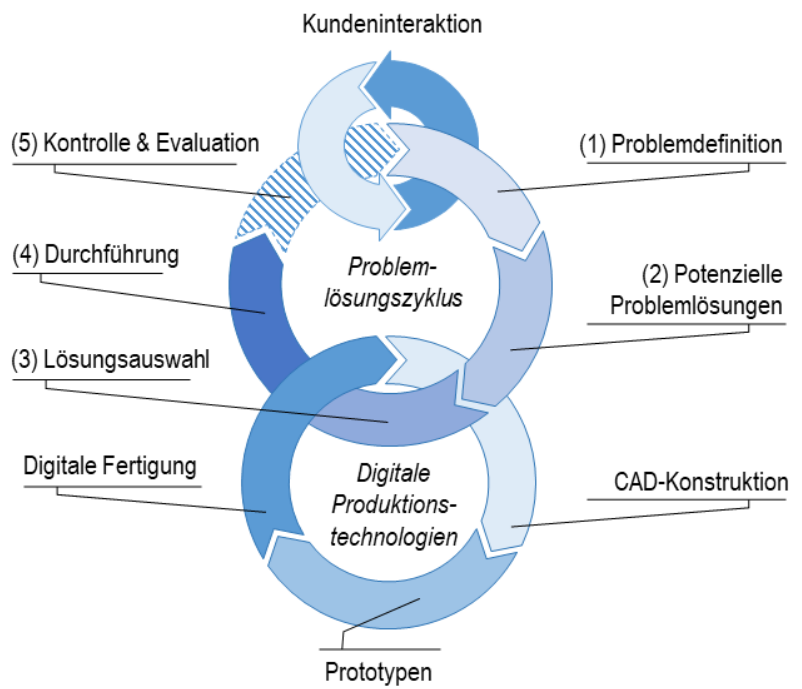

Abbildung 3: Zirkulär-iterativer Wertschöpfungsprozess in neuen Formen der digitalen urbanen Produktion

Quelle: Eigene Darstellung in Anlehnung an Stabell/Fjeldstad (1998: 424)

in zirkuläre Prozesse und iterative Feedback-Schleifen kann somit - in Erweiterung ursprünglicher Annahmen des Value-Shop-Ansatzes - als markantes Kennzeichen für digitale urbane Produktion gelten. Digitalisierung fördert dabei sowohl die fortlaufende Kundenkommunikation über digitale Medien, wie sie es auch gestattet, verschiedene Produktvarianten einfach am Computer zu entwerfen und über Rapid Prototyping per 3D-Druck zu testen, hierbei die Kundinnen/Kunden fortgesetzt am Lösungsfortschritt zu beteiligen. Für diese Kundeninteraktion schafft die Unternehmenseinbettung ins städtische Umfeld maßgebliche Vorteile und unsere Fallstudien haben die Bedeutsamkeit räumlicher Nähe für solche Beziehungen klar bestätigt.

Zweitens sind die spezifischen Lösungsansätze der digitalen Produktionstechnologien in den erweiterten Value-Shop-Zyklus der digitalen urbanen Produktion zu integrieren (unterer Bereich von Abbildung 3): Sie umfassen zum einen die kognitiv-konzeptionellen Tätigkeiten der Konstruktion mittels CAD-Systemen (klassisch bei ,etablierten' Ingenieurdienstleistern) sowie zum anderen die handwerklich-kreativen Tätigkeiten der Prototypenherstellung und des 3D-Drucks. Diese Techniken betreffen bzw. interagieren mit den Zyklus-Feldern (2) „Problemlösung“, (3) „Lösungsauswahl“ und (4) „Durchführung", wie die Verbindung der Kreisläufe in Abbildung 3 illustriert. Es kann zum mehrfachen, das heißt iterativen Durchlauf digitaler Ansätze kommen, bevor die finale 
Lösung mit dem Kunden evaluiert wird. Daher ist dieser technologiegetriebene Sub-Zyklus als eigenes Element des angepassten Value-Shop-Ansatzes zu verstehen.

Im Modell bilden somit neue digitale Produktionstechnologien das maßgebliche Scharnier zwischen kreativ-konzeptionellen und handwerklich-praktischen Tätigkeiten. Erst die Digitalisierung ermöglicht Unternehmen die Vorwärts- bzw. Rückwärtsintegration entlang der Wertekette, indem sie zuvor aufwendig manuell durchzuführende Arbeitsschritte erheblich verkürzt. Dies unterstützt dann auch den iterativ-zirkulären Charakter des Value-Shop-Problemlösungszyklus der digitalen urbanen Produktion. Weil die neuen Technologien die Produktionsgeschwindigkeiten steigern, erhöhen sie auch die Frequenz der Lösungsiterationen, was komplexere Problemstellungen bearbeitbar macht. Letztlich ist der Problemlösungszyklus als fortgesetzter Durchlauf der verschiedenen Schritte zu begreifen, deren sequenzielle Interaktion beim Wertschöpfungsprozess kaum noch eine klare Trennung der Elemente erlaubt.

Die Elemente des Problemlösungszyklus werden in der Regel nicht auf verschiedene Standorte aufgeteilt, sondern verlangen als kontinuierliche Sequenz interagierender Teilprozesse die Ausführung am selben Standort. Somit werden positive Skaleneffekte durch eine räumliche Arbeitsteilung tendenziell erschwert (Bathelt/Glückler 2018: 183). Dieser aus dem Bereich (wissensintensiver) Dienstleistungen bekannte Zusammenhang (Glückler 2007: 950) wird nun auch für Produktionsunternehmen diskutiert: Die Werkstatt der Handwerksingenieure (Typ A2) bzw. der an das Ingenieurbüro angeschlossene 3D-Drucker (Typ A1) bilden den Wirkungsort der Problemlösungs- und Wertschöpfungsprozesse ab. Somit könnte dieser Ort auch für die digitale urbane Produktion als „Wertstätte" verstanden werden, die entsprechend organisiert ist (Handke 2014: 209). Dies zeigt, dass der Value-Shop-Ansatz neben Nutzungskontexten wissensintensiver Dienstleister auch zur Erklärung neuartiger Produktionsformen wie der digitalen urbanen Produktion geeignet ist. Denn nicht nur die organisationale Zusammenführung von Vorproduktion und Produktion, sondern erst deren räumliche KoLokation unter Nutzung digitalisierter Fertigungsverfahren bildet den spezifischen Problemlösungskontext der hybriden digitalen urbanen Produktion.

Für die dargestellten Typen hybrider digitaler urbaner Produktion ist auch deshalb eine räumliche Trennung der Wertschöpfungsstufen bzw. Sub-Zyklen wenig wahrscheinlich, weil immer kleiner dimensionierte Fertigungsanlagen und zu Losgröße 1 tendierende Produktionsmengen deutlich weniger Fläche verlangen (vgl. auch Pisano/Shih 2012a: 98). Erst die Ko-Lokation des gesamten Problemlösungs- und Wertschöpfungszyklus erzeugt dessen spezifische komparative Vorteile gegenüber organisatorisch wie räumlich fragmentierten Prozessketten (vgl. auch Handke 2014 für Industrieunternehmen, die nicht dezidiert urban verortet sind). Dies unterstreichen die A1-Fallbeispiele der Ingenieurdienstleister, die trotz Einsatz von als ,ungebunden' (footloose) charakterisierbaren Technologieprozessen die räumliche Nähe zu Kundinnen und Kunden suchen. Die A2-Fallbeispiele der Handwerksunternehmen machen deutlich, dass die kreative Lösungsfindung als geradezu fluide Sequenz manuell-handwerklicher Tätigkeiten und konstruktiv-digitaler Aufgaben zu verstehen ist, deren Umsetzung mehrfach zwischen Werkstatt und Büro wechselt (gemäß des technologischen Zyklusbereichs unten in Abbildung 3). Unsere Studie bestätigt, dass Hybridität das (urban) produzierende Handwerk an der Schnittstelle von Dienstleistung und Produktion kennzeichnet (Piegeler/Spars 2019: 10).

Aus Nachfragesicht dient digitale urbane Produktion in erster Linie als Lieferant individualisierter Produkte in Nischenmärkten (De Propris/Storai 2019: 390). Dies unterscheidet die von uns identifizierten neuartigen Formen von anderen dokumentierten Beispielen, welche die urbane Herstellung von Nahrungsmitteln oder sonstigen Produkten für Endverbraucher betreffen (z. B. Lange 2017: 35). Unsere Typen digitaler urbaner Produzenten beliefern (bisher) vorwiegend - wenn auch nicht ausschließlich - Geschäftskunden (B2B-Märkte), welche hohe Anforderungen an Produkte bzw. Lösungen stellen, was bisherige Erkenntnisse zu Implikationen digitaler Produktionstechnologien bestätigt (Laplume/Petersen/ Pearce 2016: 595). Schließlich gestattet die erläuterte Konzeptualisierung, die definitorische Unschärfe des Begriffs der urbanen Produktion im Kontext von Digitalisierung bzw. Industrie 4.0 abzumildern (Stiehm 2017: 30). Die dezidiert hybride digitale urbane Produktion wird begreifbar als durch die Digitalisierung ermöglichte Form der urbanen Warenproduktion, die von zirkulär verlaufenden, problemorientierten Wertschöpfungsansätzen geprägt ist.

Verschiedene offene Aspekte der hybriden digitalen urbanen Produktion erfordern dennoch eine kritische Reflexion. Unklar bleibt bis dato, inwiefern Typen hybriddigitaler Produktion auch außerhalb von Stadtregionen auftreten können, beispielsweise im ländlichen Raum, der nach wie vor hohe Disparitäten in Bezug auf die digitale Infrastruktur aufweist (vgl. Salemink/Strijker/ Bosworth 2017: 360). Ferner bleibt offen, wie sich Urbanitätsvorteile für die hybride digitale urbane Produktion 
konkretisieren oder unterscheiden und inwiefern über die Typen unserer empirischen Untersuchung hinaus noch weitere Ausprägungsformen vorliegen. Die definitorische Unschärfe des Begriffes der (digitalen) urbanen Produktion und seine unzulängliche Operationalisierbarkeit erlauben bisher kaum eine quantitative Erfassung des Phänomens. Hierin liegen zentrale zukünftige Forschungsfelder, für die wir in diesem Beitrag mit der begrifflichen Präzisierung der hybriden digitalen urbanen Produktion eine Basis schaffen.

\section{Fazit und Ausblick}

Dieser Beitrag exploriert, wie digitale Technologien neue, hybride Formen industrieller wie handwerklicher Produktion im urbanen Raum vorantreiben. Die hierzu erarbeiteten konzeptionellen Vorschläge greifen den Value-ShopAnsatz auf (Stabell/Fjeldstad 1998: 420; Lange/Bürkner 2018a: 99) und entwickeln inn weiter, gestützt auf eigene qualitative empirische Forschungsansätze. Es zeigt sich, dass neuartige Typen der digitalen urbanen Produktion aufkommen, die in hohem Maße von der Einbettung ins urbane Umfeld profitieren. Die Wertschöpfungslogiken dieser neuen Unternehmenstypen sind nicht linear ausgerichtet, sondern - in Anlehnung an den Value-ShopAnsatz - zirkulär-iterativ und problemzentriert. Warenproduktion und Dienstleistungen werden - im Sinne von symbiotic recoupling ${ }^{3}$ (De Propris/Storai 2019: 390) - in hybrider Weise verknüpft angeboten, bei Übernahme zunehmend komplizierter Aufgaben. Typisch ist dabei, dass die Betriebe handwerklich-praktische Tätigkeiten mittels digitaler Tools und Anwendungen konstruktiv mit kreativ-konzeptionellen Ingenieurdienstleistungen kombinieren. Ein Standort im Stadtraum erweist sich dabei als großer Vorteil, weil dort die benötigten hoch qualifizierten Fachkräfte sowie die Nachfrager für die angebotenen spezifischen Lösungen konzentriert sind, die auch untereinander von der Ko-Lokation profitieren. So kann gleichfalls die Einbettung ins städtische Innovationssystem die aufkommenden digitalen urbanen Produzenten fördern.

Dass die hybride Zusammenführung von Produktion und Dienstleistung vor allem in Städten geschieht, lässt sich aus der Angebotsperspektive urbaner Produzenten wie aus der Nachfrageperspektive heraus begründen.

3 Darunter wird eine funktionale wie räumliche Wiederverknüpfung von Produktions- und Dienstleistungsaktivitäten verstanden, die vormals getrennt waren (De Propris/Storai 2019: 390).
Anbieter, das heißt digital unterstützte Ingenieurdienstleister und Handwerksunternehmen, profitieren im städtischen Raum insbesondere vom reichhaltigen Arbeitsmarkt (Stiehm 2017: 73) und Innovationssystem (Florida/ Adler/Mellander 2017: 93; Reynolds 2017: 26; Reynolds/ Uygun 2018: 178), benötigen sogar zwingend diese Standortfaktoren. Unsere Untersuchungen bestätigen, dass für die anspruchsvollen, zirkulären Wertschöpfungsprozesse digitaler urbaner Produktion, die nicht nur die kreativ-konzeptionellen Kenntnisse eines Ingenieurs, sondern auch das manuelle produktionsbezogene Geschick eines Handwerkers verlangen, hoch spezialisierte und qualifizierte Fachkräfte essenziell sind.

Aus den besonderen Standortanforderungen digitaler urbaner Produktion ergeben sich wichtige Implikationen für die Praxis von Stadtplanung und Wirtschaftsförderung. Dies gilt insbesondere hinsichtlich des Gewerbeflächenmanagements, das beispielsweise die temporäre Zwischennutzung brachliegender Industrieund Büroimmobilien beobachtet. Dies schafft Potenziale zur ökonomischen Aufwertung von Stadtquartieren und bietet Unternehmen den Raum für die Erprobung innovativer Geschäftspraktiken (für weitere Implikationen vgl. auch Mühl/Busch/Fromhold-Eisebith et al. 2019: 77 f.). Für romantisierende Vorstellungen einer Re-Integration von komplexen Wertschöpfungsketten an Orten hoch qualifizierter Produktion in den traditionellen Zentren ist dennoch wenig Platz. Schließlich leben einige der Handwerksbetriebe und Ingenieurdienstleister, die wir untersucht haben, besonders auch von Aufträgen größerer Firmen, die ihrerseits diese Tätigkeiten zu flexibleren Anbietern hin outsourcen. Insofern wird die kostengetriebene Auslagerungstendenz aus sozialpartnerschaftlich geregelten Bereichen hin zu diesbezüglich weniger regulierten Kleinunternehmen weiter fortgesetzt. Für die lokalen Stakeholder im städtischen Raum, wie Wirtschaftsförderungen, Kammern, Gewerkschaften, erscheint es insofern lohnend, die hier vorgestellten neuen Formen der Produktion im digitalen Zeitalter aufmerksam zu betrachten.

Danksagung: Wir möchten uns beim Forschungsinstitut für gesellschaftliche Weiterentwicklung (FGW) für die finanzielle Förderung aus Mitteln des Ministeriums für Kultur und Wissenschaft des Landes Nordrhein-Westfalen bedanken (Förderzeitraum 2016-2018). Insbesondere gilt unser Dank dort Prof. Dr. Hartmut Hirsch-Kreinsen und Anemari Karačić für die stets gute Zusammenarbeit. Für kritische und hilfreiche Kommentare auf eine frühere Version des Manuskripts danken wir zudem zwei anonymen Gutachtern und der Schriftleitung dieser Zeitschrift 
sowie den Teilnehmenden der Jahrestagung 2019 des Arbeitskreises Industriegeographie.

\section{Literatur}

Adam, H. (2001): Reale Orte in digitalen Netzen. In: Raumforschung und Raumordnung 59, 2-3, 166-172. doi: 10.1007/BF03184351

Bathelt, H.; Glückler, J. (2018): Relational Research Design in Economic Geography. In: Clark, G. L.; Feldman, M. P.; Gertler, M. S.; Wójcik, D. (Hrsg.): The New Oxford Handbook of Economic Geography. Oxford, 179-195. doi: 10.1093/ oxfordhb/9780198755609.013.46

Birch, K.; MacKinnon, D.; Cumbers, A. (2010): Old Industrial Regions in Europe: A Comparative Assessment of Economic Performance. In: Regional Studies 44, 1, 35-53. doi: 10.1080/00343400802195147

Buciuni, G.; Pisano, G. (2018): Knowledge integrators and the survival of manufacturing clusters. In: Journal of Economic Geography 18, 5, 1069-1089. doi: 10.1093/jeg/lby035

De Propris, L.; Storai, D. (2019): Servitizing industrial regions. In: Regional Studies 53, 3, 388-397. doi: 10.1080/00343404.2018.1538553

Ernst, D. (1985): Automation and the Worldwide Restructuring of the Electronics Industry: Strategic Implications for Developing Countries. In: World Development 13, 3, 333-352. doi: 10.1016/0305-750X(85)90134-2

Florida, R.; Adler, P.; Mellander, C. (2017): The city as innovation machine. In: Regional Studies 51, 1, 86-96. doi: 10.1080/00343404.2016.1255324

Fromhold-Eisebith, M. (2017): Cyber-Physical Systems in Smart Cities - Mastering Technological, Economic, and Social Challenges. In: Song, H.; Srinivasan, R.; Sookoor, T.; Jeschke, S. (Hrsg.): Smart Cities: Foundations, Principles and Applications. Hoboken, 1-22.

Fuchs, M.; Fromhold-Eisebith, M.; Busch, H.-C.; Mühl, C. (2017): ,Urbane Produktion؛ Dynamisierung stadtregionaler Arbeitsmärkte durch Digitalisierung und Industrie 4.0? Köln. = Working Paper des Wirtschafts- und Sozialgeographischen Instituts 2017-01.

Gertler, M. S. (2003): Tacit knowledge and the economic geography of context, or The undefinable tacitness of being (there). In: Journal of Economic Geography 3, 1, 75-99.

Glückler, J. (2007): Geography of Reputation: The City as Locus of Business Opportunity. In: Regional Studies 41, 7, 949-961. doi: 10.1080/00343400601145194

Gornig, M.; Werwatz, A. (2018): Anzeichen für eine Reurbanisierung der Industrie. In: DIW Wochenbericht 85, 47, 1006-1011. doi: 10.18723/diw_wb:2018-47-2

Gress, D. R.; Kalafsky, R. V. (2015): Geographies of production in 3D: Theoretical and research implications stemming from additive manufacturing. In: Geoforum 60, 43-52. doi: 10.1016/j. geoforum.2015.01.003

Hamm, R. (2013): Verflechtungen zwischen Industrie und unternehmensorientierten Dienstleistungen - eine empirische Analyse für das Rheinland. In: Raumforschung und Raumordnung 71, 2, 87-98. doi: 10.1007/s13147-013-0214-2
Handke, M. (2014): Die Verortung der Wertschöpfung durch Dienstleistungen. Die Organisation von Wertstätten in der Kunststoff verarbeitenden Industrie Mittelfrankens. In: Pechlaner, H.; Doepfer, B. C. (Hrsg.): Wertschöpfungskompetenz und Unternehmertum. Rahmenbedingungen für Entrepreneurship und Innovation in Regionen. Wiesbaden, 197-252. doi: 10.1007/978-3-65800923-6

Henckel, D.; Nopper, E.; Rauch, N. (1984): Informationstechnologie und Stadtentwicklung. Stuttgart. = Schriften des Deutschen Instituts für Urbanistik 71.

Hirsch-Kreinsen, H. (2016): Digitization of industrial work: development paths and prospects. In: Journal for Labour Market Research 49, 1, 1-14. doi: 10.1007/s12651-016-0200-6

Hoffman, K. (1985): Clothing, Chips and Competitive Advantage. The Impact of Microelectronics on Trade and Production in the Garment Industry. In: World Development 13, 3, 371-392. doi: 10.1016/0305-750X(85)90136-6

Ivarsson, I.; Alvstam, C.; Vahlne, J.-E. (2017): Global technology development by colocating R\&D and manufacturing: the case of Swedish manufacturing MNEs. In: Industrial and Corporate Change 26, 1, 149-168. doi: 10.1093/icc/dtw018

Juraschek, M.; Vossen, B.; Hoffschröer, H.; Reicher, C.; Herrmann, C. (2018): Urbane Produktion: Ökotone als Analogie für eine nachhaltige Wertschöpfung in Städten. In: Redlich, T.; Moritz, M.; Wulfsberg, J. P. (Hrsg.): Interdisziplinäre Perspektiven zur Zukunft der Wertschöpfung. Wiesbaden, 195-207. doi: 10.1007/978-3-658-20265-1_15

Kagermann, H.; Anderl, R.; Gausemeier, J.; Schuh, G.; Wahlster, W. (2016): Industrie $4.0 \mathrm{im}$ globalen Kontext: Strategien der Zusammenarbeit mit internationalen Partnern. München.

Kempermann, H.; Lichtblau, K. (2012): Definition und Messung von hybrider Wertschöpfung. In: IW Trends 1, 1-20.

Läpple, D. (2016): Produktion zurück in die Stadt. Ein Plädoyer. In: Bauwelt 35, 22-29.

Lafuente, E.; Vaillant, Y.; Vendrell-Herrero, F. (2019): Territorial servitization and the manufacturing renaissance in knowledgebased economies. In: Regional Studies 53, 3, 313-319. doi: 10.1080/00343404.2018.1542670

Lange, B. (2017): Neue Formen des Produzierens in der Stadtregion. In: Nachrichten der ARL 47, 4, 33-36.

Lange, B.; Bürkner, H.-J. (2018a): Flexible value creation: Conceptual prerequisites and empirical explorations in open workshops. In: Geoforum 88, 96-104. doi: 10.1016/j. geoforum.2017.11.020

Lange, B.; Bürkner, H.-J. (2018b): Open workshops as sites of innovative socio-economic practices: approaching urban postgrowth by assemblage theory. In: Local Environment 23, 7, 680-696. doi: 10.1080/13549839.2017.1418305

Laplume, A. O.; Petersen, B.; Pearce, J. M. (2016): Global value chains from a $3 \mathrm{D}$ printing perspective. In: Journal of International Business Studies 47, 5, 595-609. doi: 10.1057/ jibs.2015.47

Leamer, E. E.; Storper, M. (2001): The Economic Geography of the Internet Age. In: Journal of International Business Studies 32, 4, 641-665. doi: 10.1057/palgrave.jibs.84909988

Leborgne, D.; Lipietz, A. (1988): New Technologies, New Modes of Regulation: Some Spatial Implications. In: Environment and Planning D 6, 3, 263-280. doi: 10.1068/d060263 
Lentes, J. (2017): Urbane Produktion. In: Spath, D.; Westkämper, E.; Bullinger, H.-J.; Warnecke, H.-J. (Hrsg.): Neue Entwicklungen in der Unternehmensorganisation. Berlin, 45-55. doi: http:// dx.doi.org/10.1007/978-3-662-55426-5

Matt, D. T.; Orzes, G.; Rauch, E.; Dallasega, P. (2018): Urban production - A socially sustainable factory concept to overcome shortcomings of qualified workers in smart SMEs. In: Computers and Industrial Engineering 139, 105384, 1-10. doi: 10.1016/j.cie.2018.08.035.

Mattissek, A.; Pfaffenbach, C.; Reuber, P. (2013): Methoden der empirischen Humangeographie. Braunschweig.

Mayring, P. (2000): Qualitative Content Analysis. In: Qualitative Social Research 1, 2, 1-10.

Moriset, B.; Malecki, E. J. (2009): Organization versus Space: The Paradoxical Geographies of the Digital Economy. In: Geography Compass 3, 1, 256-274. doi: 10.1111/j.17498198.2008.00203.x

Mühl, C.; Busch, H.-C.; Fromhold-Eisebith, M.; Fuchs, M. (2019): Urbane Produktion - Dynamisierung stadtregionaler Arbeitsmärkte durch Digitalisierung und Industrie 4.0? Düsseldorf. = FGW-Impuls Digitalisierung von Arbeit 14.

Musil, R.; Eder, J. (2017): Wozu räumliche Nähe in der urbanen Wissensökonomie? Eine geostatistische Analyse Wiener Forschungscluster. In: Raumforschung und Raumordnung | Spatial Research and Planning 75, 2, 93-107. doi: 10.1007 | s13147-016-0458-8

Piegeler, M.; Spars, G. (2019): Urbane Produktion - Konzept und Messung. Wuppertal.

Pisano, G. P.; Shih, W. C. (2012a): Does America Really Need Manufacturing? In: Harvard Business Review 97, 3, 94-102.

Pisano, G. P.; Shih, W. C. (2012b): Producing Prosperity: Why America Needs a Manufacturing Renaissance. Boston.

Reynolds, E. B. (2017): Innovation and Production: Advanced Manufacturing Technologies, Trends and Implications for US Cities and Regions. In: Built Environment 43, 1, 25-43. doi: 10.2148/benv.63.3.25

Reynolds, E.B.; Uygun, Y. (2018): Strengthening advanced manufacturing innovation ecosystems: The case of Massachusetts. In: Technological Forecasting and Social Change 136, 178-191. doi: 10.1016/j.techfore.2017.06.003

Salemink, K.; Strijker, D.; Bosworth, G. (2017): Rural development in the digital age: A systematic literature review on unequal ICT availability, adoption, and use in rural areas. In: Journal of Rural Studies 54, 360-371. doi: 10.1016/j.jrurstud.2015.09.001

Spath, D. (2014): Urbane Produktion. In: Weinert, K.; Beckmann, K. J.; Encarnacao, J. L.; Herzog, O.; Höcker, H.; Kuhn, A.; Mühlhäuser, M.; Schober, O.; Spath, D.; Thoma, K. (Hrsg.): Stadt der Zukunft - Strategieelemente einer nachhaltigen Stadtentwicklung. München, 61-71.

Stabell, C. B.; Fjeldstad, Ø. D. (1998): Configuring value for competitive advantage: on chains, shops, and networks. In: Strategic Management Journal 19, 5, 413-437. doi: 10.1002/(SICI)1097-0266(199805)19:5\%3C413::AIDSMJ946\%3E3.0.CO;2-C

Stiehm, S. D. (2017): Gestaltungsparameter für die (Re-)Integration von Produktion in den urbanen Raum im Kontext von Industrie 4.0. Aachen.

van Assche, A. (2017): Global Value Chains and Innovation. In: Bathelt, H.; Cohendet, P.; Henn, S.; Simon, L. (Hrsg.): The
Elgar Companion to Innovation and Knowledge Creation. Cheltenham, 739-751.

van Winden, W.; van den Berg, L.; Carvalho, L.; van Tuijl, E. (2011):

Manufacturing in the new urban economy. London. 